\title{
The Relationship between the Sialic Acid Concentrations in the Serum and Whole Saliva in Rats with Naturally Occurring Gingivitis
}

\author{
Mitsuko Shinohara, Kiyoshi Ohura, Keigo Ogata, Hiroaki Inoue, Toshio Miyata and Mitsushi Yoshioka \\ Department of Pharmacology, Osaka Dental University, 5-3I Otemae I-chome, Chuo-ku, Osaka 540, Japan \\ Received September 16, 1993 Accepted November 27, 1993
}

\begin{abstract}
Pocket probing depth was correlated with the amount of salivary sialic acid in pilocarpinestimulated saliva in ODU plaque-susceptible rats (ODUS/Odu) $(r=0.657, \mathrm{P}<0.01)$, but not with the content of serum sialic acid. There was no difference in the amount of serum sialic acid content between ODUS/Odu and plaque-resistant rats. These results suggest that the amount of sialic acid in the saliva can be a useful index of the severity of periodontal disease.
\end{abstract}

Keywords: Sialic acid, Gingivitis, Saliva

It is generally accepted that the oral environment is influenced by the composition of saliva, as it affects both oral tissue and flora. Recently, there have been many reports indicating that serum sialic acid increases when inflammation occurs $(1-3)$.

Since 1972, ODU plaque-susceptible rats (ODUS/ Odu), which were derived from the Wistar-Kyoto strain, have been bred in our laboratory. We previously reported that these rats serve as a very suitable experimental animal model for studying gingivitis (4-11). The relationship between the amount of plaque and the pocket probing depth (PPD) showed a high positive correlation (4), so that for ODUS/Odu, the severity of gingivitis was judged from the PPD. We therefore investigated the relationship between the concentration of sialic acid in the serum and whole saliva and the degree of periodontal disease. Twenty-nine male ODUS/Odu and 14 male plaque-resistant rats (Res), all approximately 5 months of age (body weight: $350-400 \mathrm{~g}$ ), were used. PPD for ODUS/Odu animals were measured by the method described previously (5). The animals were anesthetized with sodium pentobarbital (Abbott Laboratories, North Chicago, IL, USA) intraperitoneally $(50 \mathrm{mg} / \mathrm{kg}$ ). Measurements were taken at the center of each mandibular incisor, after the removal of plaque. Plaque was collected from the cervical region of the lower incisors using a scaler. A tracheal cannula was inserted. As a stimulant, pilocarpine hydrochloride (Nacalai Tesque, Kyoto; $8 \mathrm{mg} / \mathrm{kg}$ ) was injected intraperitoneally, and the saliva was collected for $40 \mathrm{~min}$ by the method of Navia (12). Blood samples were obtained from a femoral vein. The sialic acid ( $N$-acetylneuraminic acid) content was analyzed by using sialic acid test reagents (Kyokuto-Seiyaku Inc., Tokyo). This analysis is an enzyme method that uses neuraminidase. There was no significant difference in serum sialic acid concentration between ODUS/Odu and Res. Also, in ODUS/Odu, there was no correlation between the sialic acid concentration in the serum and PPD (Fig. 1). There was a significant difference in salivary sialic acid concentration between ODUS/Odu and Res $(P<0.001)$ and also a high positive correlation $(\mathrm{Y}=0.018 \mathrm{X}+0.040, \mathrm{r}=0.657, \mathrm{P}<0.01)$ between the sialic acid concentration in pilocarpine-stimulated whole saliva and the PPD in ODUS/Odu (Fig. 2).

Periodontal diseases are characterized by a chronic inflammatory reaction of the periodontal membrane and of the tissues surrounding the gingiva as a result of persistent local stimulation by the bacteria in dental plaque and by their metabolites. In addition, saliva plays an important role in the onset and course of inflammation, and in various infections in the oral cavity.

Sialic acid is a general term for a derivative of neuraminic acid, a compound with 9 carbon atoms, a carboxyl, carbonyl, hydroxyl and amino group, and is found as a component of glycoproteins, mainly of the $\alpha$ - and $\beta$ globulin fractions. In vivo, serum sialic acid is contained in glycoproteins and glycolipids, which are found in body fluid, tissue, secretions, and various cell membranes, and it exists at the terminal of sugar chains. Sialic acid has various biological actions. For example, it increases the viscosity of a glycoprotein solution, and it binds and 


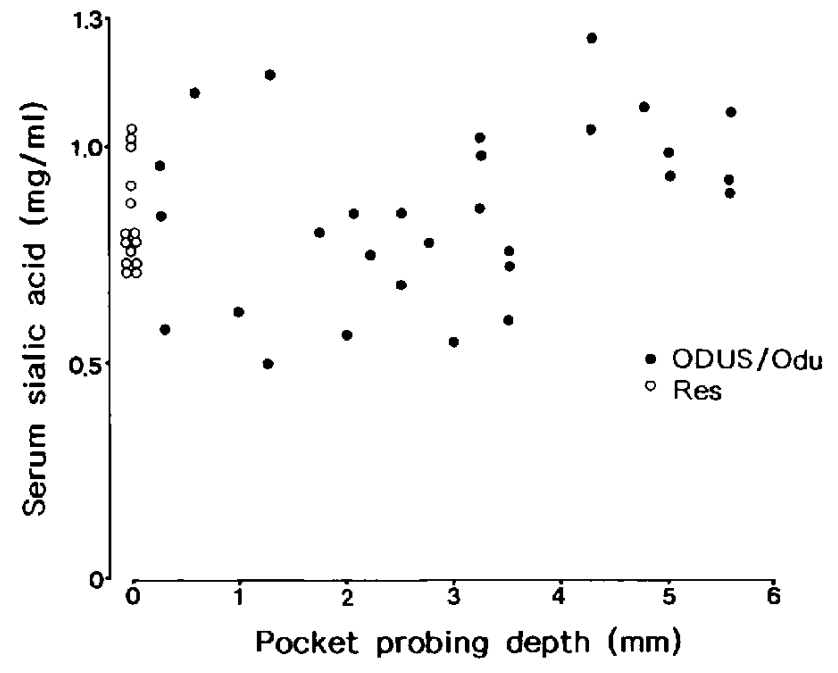

Fig. 1. Relationship between the sialic acid concentration in serum and the pocket probing depth in ODU plaque-susceptible rats (ODUS/Odu) and plaque-resistant rats (Res).

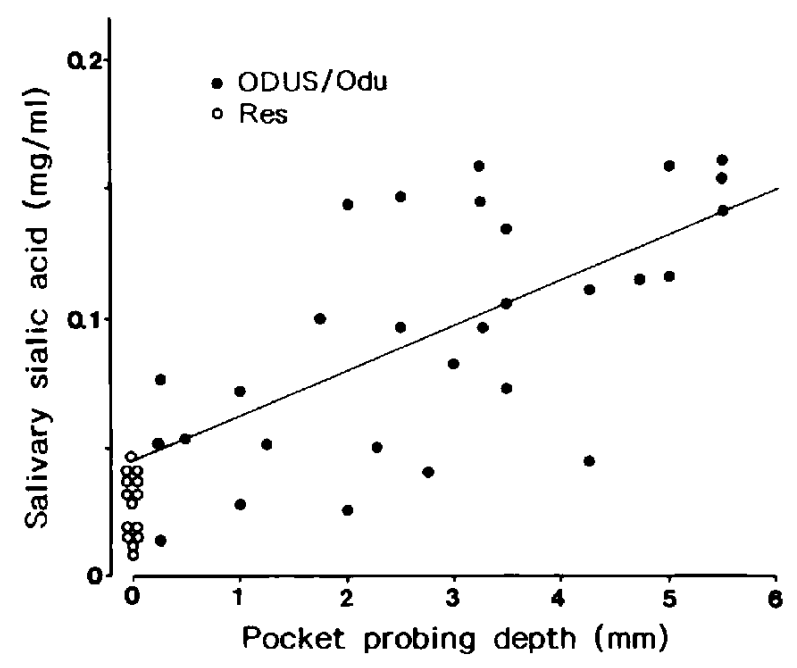

Fig. 2. Relationship between ODU plaque-susceptible rats (ODUS/Odu) and plaque-resistant rats (Res) in the salivary sialic acid concentration $(P<0.001)$ and that between the sialic acid concentration in pilocarpine-stimulated saliva and pocket probing depth in ODUS/Odu ( $\mathrm{Y}=0.018 \mathrm{X}+0.040, \mathrm{r}=0.657, \mathrm{P}<0.01)$.

transports molecules and viruses. The serum sialic acid concentration has been clinically demonstrated to increase in association with inflammatory diseases such as rheumatoid arthritis and pneumonia, and in malignant tumors (1-3).

In the present experiment, we investigated the relationships between the severity of gingivitis and the serum and salivary sialic acid concentration in ODUS/Odu, now in the 52nd generation. There was a highly positive correlation between the amount of plaque and the PPD. There- fore, the PPD was used as an index for evaluating the severity of gingivitis. The serum sialic acid concentration was then determined in both ODUS/Odu and Res. No difference in the concentration of serum sialic acid was found between ODUS/Odu and Res. There was no correlation between the severity of gingivitis and the sialic acid concentration even among ODUS/Odu. Iwai et al. (13) determined the serum sialic acid concentration in three groups of patients with acute bacterial infection of the oral cavity. These investigators reported that the sialic acid concentration increased with the severity of inflammatory symptoms compared to a group of patients with cellulitis and another diseases with relatively mild symptoms, such as maxillary ostitis, perimaxillary inflammation, pericoronitis of the wisdom teeth, and apical periodontitis.

Gingivitis is considered a local inflammation, therefore there was no difference in the serum sialic acid concentration in ODUS/Odu and Res. Kou et al. (14) studied serum sialic acid concentrations in patients with cancer of the oral cavity. The investigators compared the levels in a group with severe disease, a group with mild disease and a healthy group, and they found significantly higher levels in the group with severe disease. They found that the sialic acid concentration within one month after surgery was much higher than it was before the surgery. Further investigation revealed that the level decreased one month or more after the surgery, and it was much lower 3 months after surgery than it was before surgery was performed. Ito et al. (15) studied the relationship between the total salivary sialic acid concentration and the clinical symptoms of periodontal disease. According to their report, there was a correlation $(P<0.05)$ between the severity of gingivitis and the salivary sialic acid concentration. The salivary sialic acid concentration in the affected group was significantly higher than that in the healthy group $(\mathrm{P}<0.05)$.

In the present study, there was a correlation between the severity and the salivary sialic acid concentration. These results suggest that the total salivary sialic acid concentration is a useful parameter for determining the severity and course of periodontal diseases.

\section{REFERENCES}

1 Krolikowski, F.J., Reuter, K., Waalkes, T.P., Sieber, S.M. and Adamson, R.H.: Serum sialic acid levels in lung cancer patients. Pharmacology 14, 41-51 (1976)

2 Silver, H.K., Ranger, D.M. and Morton, D.L.: Serum sialic acid elevation in malignant melanoma patients. Cancer 41, $1497-1499$ (1978)

3 Hogen-Ryan, A., Fennelly, J.J., Jones, M., Cantwell, B. and Duffy, M.J.: Serum sialic acid and cea concentration in human breast cancer. Br. J. Cancer 41, 587-592 (1980) 
4 Takai, N., Shinohara, M., Yoshida, Y., Ohura, K., Mori, M. and Kakudo, Y.: Effect of streptozotocin diabetes on gingivitis in plaque-susceptible rats. J. Dent. Res. 65, 49-52 (1986)

5 Takai, N., Shinohara, M., Yoshida, Y., Mori, M. and Kakudo, Y.: Studies on saliva in ODU plaque-susceptible rats having experimental gingivitis: II. IgA in the saliva. J. Periodont. Res. 20, 317-320 (1985)

6 Ohura, K., Tani, A., Shinohara, M., Fujimoto, H., Sagawa, H. and Mori. M.: The relationship between serum immunoglobulin levels and pocket probing depth in rats with naturally occurring gingivitis. In Recent Advances in Clinical Periodontology, Edited by Ishikawa, J., Kawasaki, H., Ikeda, K. and Hasegawa, K., pp. 641-644, Elsevier Science Publishing Co., New York (1988)

7 Ohura, K., Shinohara, M., Ogata, K., Nishiyama, A. and Mori, M.: Leucocyte function in rats with naturally occurring gingivitis. Arch. Oral Biol. 35, 185S-187S (1990)

8 Shinohara, M., Takai, N., Ohura, K., Yoshida, Y., Mori, M. and Kakudo, Y.: The $\mathrm{pH}$ in periodontal pocket of ODU plaquesusceptible rats having experimental gingivitis. Jpn. J. Oral Biol. 26, 525-527 (1984)

9 Shinohara, M., Ohura, K., Ogata, K. and Mori, M.: A new rat strain with naturally occurring gingivitis. Rat News Lett. 24, 4-6 (1991)

10 Fujimoto, H., Ohura, K., Shinohara, M., Nishiyama, A.,
Kumon, Y., Nishida, E. and Mori, M.: Macrophage chemotaxis in rats with naturally occurring gingivitis. In Recent Advances in Clinical Periodontology, Edited by Ishikawa, J., Kawasaki, H., Ikeda, K. and Hasegawa, K., pp. 645-648, Elsevier Science Publishing Co., New York (1988)

11 Konakajima, Y., Tani, A., Ohura, K., Shinohara, M., Ogata, K., Mori, M. and Sagawa, H.: Humoral immune response in experimental gingivitis rats. Arch. Oral Biol. Supp. 181s-183s (1990)

12 Navia, J.M.: The oral environment in experimental animals. Saliva and salivary glands. In Animal Model in Dental Research, pp. 225-254, The University of Alabama Press, Birmingham (1977)

13 Iwai, Y., Kou, M., Yagami, K., Iwase, M., Ito, S., Nishimura, A. and Nagumo, M.: Serum sialic acid in acute oral bacterial infections. Jpn. J. Oral Maxillofac. Surg. 32, 380-385 (1986) (Abs. in English)

14 Kou, M., Iwai, Y., Kanemoto, K., Hori, S., Yoshiya, M. and Nagumo, M.: Serum sialic acid in patients with oral cancer. Jpn. J. Oral Maxillofac. Surg. 32, 16-21 (1986) (Abs. in English)

15 Ito, F., Yamada, S., Omori, S., Yamada, H. and Nakashizuka, T.: The relationship between the sialic acid contents in saliva and the clinical findings. J. Jpn. Assoc. Periodont. 17, 110-114 (1975) (Abs. in English) 